DOI: https://doi.org/10.46296/ig.v4i7.0020

\title{
DISEÑO CONCEPTUAL DE UN BANCO DE PRUEBAS PARA LA EVALUACIÓN DE LA CALIDAD DE CIERRE EN LATAS DE CONSERVA
}

\section{CONCEPTUAL DESIGN OF A TEST BENCH FOR THE EVALUATION OF THE QUALITY OF CLOSURE IN TIN CANS}

\author{
Tello-Macías Pablo Rafael ${ }^{1 *}$; Herrera-Suárez Miguel ${ }^{2}$ \\ ${ }^{1}$ Instituto de Posgrado de la Universidad Técnica de Manabí, UTM. Portoviejo, \\ Ecuador. https://orcid.org/0000-0002-2936-2692 \\ ${ }^{2}$ Escuela de Mecánica, Facultad de Ciencias Matemáticas, Físicas, y Químicas, \\ Universidad Técnica de Manabí, UTM. Portoviejo, Ecuador. https://orcid.org/0000- \\ 0003-4567-5872
}

*Correo: ptello3206@utm.edu.ec

\begin{abstract}
Resumen
El estudio de la incidencia del desgaste y deformación de los elementos de cierre en las condiciones de operación de la industria es complejo, por lo que es necesario su investigación en las condiciones controladas de los bancos de pruebas. El objetivo del trabajo es realizar un diseño conceptual de un banco de pruebas para la evaluación de la calidad de cierre en latas de conserva circulares que son generalmente utilizadas por empresas dedicadas a la producción de conservas de atún. A partir de un diseño conceptual se estructura una variante de diseño funcional, segura y económica para el banco de pruebas, constituida por una transmisión de potencia por banda en V. Complementariamente se realizó un análisis de resistencia de los elementos de cierre, la selección de rodamiento y análisis de costo. De los resultados del análisis de resistencia, las tensiones máximas en el mandril se distribuyen en el labio que comprime la lata, para las rulinas las deformaciones máximas son de $1,75 \cdot 10^{\wedge}(-6) \mathrm{mm}$ en la sección de los perfiles de cierre. El costo de fabricación es 827,87 USD al considerar la adquisición de materiales nuevos. El prototipo permite realizar el proceso de doble cierre de manera correcta y sencilla.
\end{abstract}

Palabras clave: Diseño conceptual; costura doble; comida enlatada; hojalata.

\begin{abstract}
The study of the incidence of the wear and deformation of the closing elements in the operation conditions of the industry is complex, so it is necessary its investigation in the controlled conditions of the test banks. The objective of the work is to carry out a conceptual design of a test bank for the evaluation of the closing quality in circular cans that are generally used by companies dedicated to the production of canned tuna. From a conceptual design, a functional, safe and economic design variant for the test bench is structured, consisting of a power transmission by Vband. Complementarily, a resistance analysis of the closing elements, the selection of bearings and cost analysis were carried out. From the results of the strength analysis, the maximum stresses in the mandrel are distributed on the lip that compresses the can, for the rolls the maximum deformations are $1,75 \cdot 10^{\wedge}(-6) \mathrm{mm}$ in the section of the seal profiles. The manufacturing cost is 827,87 USD when considering the acquisition of new materials. The prototype allows to carry out the process of double closing in a correct and simple way.
\end{abstract}

Keywords: Concept design; double stitching; canned food; tin.

Información del manuscrito:

Fecha de recepción: 01 de diciembre de 2020.

Fecha de aceptación: 08 de enero de 2021.

Fecha de publicación: 11 de enero de 2021. 


\section{Introducción}

En el Ecuador, la pesca artesanal forma parte del sustento económico para un gran número de familias y cooperativas [1], sin embargo, los recursos pesqueros alcancen su mayor potencial al ser procesados y exportados como alimento por fábricas distribuidas en las zonas costeras del país, principalmente en las provincias de Manabí y Guayas [2-3]. La captura de atún aleta amarilla (Thunnus albacares) en el territorio, comienza en el año 1952, con la industrialización de la especie en el puerto de Manta, actualmente la actividad se encuentra regulada a través de organismo de control por parte del país y se basa en resoluciones elaboradas por la Comisión Interamericana del Atún Tropical, [4]. Para el año 2014, se contabilizó un total de 27 empresas cuya finalidad es la producción de conserva de atún en latas como producto principal [5].

Para la producción de conservas de atún en latas como un producto terminado y comercial a nivel nacional e internacional se requiere de una línea de procesos bien estructurada, donde se destaca la cocción de la materia prima, el enlatado y el esterilizado [5-8]. Al hablar de alimentos procesados, se debe de tener en cuenta que los alimentos originales tendrán ciertas diferencias con respecto al producto envasado, por ejemplo, el sabor, el color y la textura, sin embargo, esta variante presenta características significativas como un intervalo de consumo más prolongado [9-10].

El proceso de enlatado o sellado de latas se define como un acoplamiento hermético del conjunto recipiente y tapa, este proceso es el encargado de determinar el tiempo de vida útil del contenido, generalmente el material utilizado para la conformación de recipiente se le denomina "hojalata" con espesores relativamente pequeños [11]. El contenido (pescado procesado) no puede entrar en contacto directo con el metal, por lo que se utilizan revestimientos orgánicos (lacas), adicionalmente en casos específicos se adiciona un esmalte blanco en el interior en los extremos [9-12-13]. Algunas de las propiedades relevantes de estos revestimientos son, la inercia química y la capacidad de soportar temperaturas de esterilización. 
El doble cierre se compone de un total de cinco dobleces de la hojalata que parten de las pestañas de la tapa y envase, esta operación se lleva a cabo a través de máquinas cerradoras en dos operaciones [14]. La primera operación (engargolado), produce una curvatura en ambas pestañas resultando un entrelazamiento entre ellas. Al ejercer muy poca presión en la primera operación no se obtendrá el enganche correcto, y este se encontrará alejado del cuerpo de la lata. La segunda operación (planchado), presiona la unión de ambas pestañas y permite que el compuesto sellante ocupe todas las cavidades del cierre [15-16]. Al aplicar una elevada presión en esta operación, se puede producir un desenganche o que ambas pestañas resbalen entre sí, dando paso a filtraciones.

Los defectos en el proceso de doble cierre pueden ser muy variados, se destacan problemas como picos en el engargolado, rebaba pronunciada, cierre incompleto, desigualdades por mala alineación, gancho de tapa corto y largo [17-18]. Algunos de los defectos pueden ser visualmente apreciables, sin embargo, existen técnicas como la inspección por rayos $\mathrm{x}$ e imagen digitalizada que permite tener un análisis más profundo de la calidad de cierre presente en las latas analizadas [1920]. Considerando que existen muchos factores que pueden afectar el cierre doble de las latas, se decide realizar el presente trabajo que tiene como objetivo el diseño conceptual de un banco de pruebas para la evaluación de la calidad de cierre en latas.

\section{Metodología}

El desarrollo de la investigación se realizó en dos etapas que contempla el diseño conceptual y construcción del banco de pruebas: en primera instancia se obtuvo bibliografía relevante referente a características $y$ funciones de maquinaria especializada para el cierre de latas, posteriormente se procedió con la elaboración del modelo virtual del banco de pruebas, en esta etapa se utiliza el software SolidWorks $\AA^{\circledR} 2020$ (Student Edition). El proceso de construcción se llevó a cabo en los talleres de elaboración mecánica de la Universidad Técnica de Manabí. 


\section{Diseño conceptual del banco de pruebas}

Se recolecta información sobre funciones, capacidad y características de las máquinas cerradoras, y a partir de la metodología del despliegue de la función de calidad o QFD, [21-22], se obtiene una variante de diseño para el prototipo de banco de prueba.

\section{Cálculo de la transmisión de potencia}

En base a la disponibilidad de espacio, aspectos funcionales y económico se considera el diseño de una trasmisión de potencia por banda "V", para la transmisión se calcula la potencia de diseño, selección del órgano motriz, cálculo de tamaños de poleas, distancia entre centros, entre otros. Los parámetros de diseño siguen la metodología de diseño de elementos de máquinas por [23].

\section{Perfiles para la operación de cierre}

La primera operación presenta un perfil mucho más profundo y estrecho (Figura 1a), su función principal radica en enrollar la hojalata de ambas pestañas, este cierre aún no es hermético. El perfil para el planchado es más alto y menos profundo en comparación a la primera operación (Figura 1b), la finalidad de esta operación es presionar y planchar el cierre, con lo cual se logra distribuir de manera apropiada el sellado evitando así filtración y pérdidas de líquidos o gases. Los principales parámetros geométricos de la rulina de primera operación, segunda operación y mandril se muestran a continuación, estas dimensiones sirven de referencia para el desarrollo de los modelos virtuales (Tabla 1).
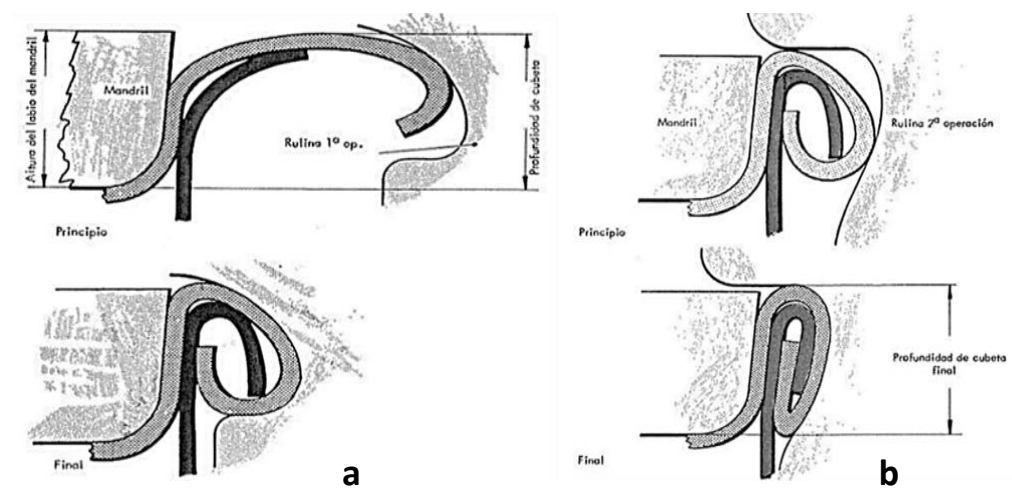

Figura 1. Primera operación (a) y segunda operación (b), [24]. 
Tabla 1. Parámetros geométricos de los elementos para el proceso de cierre.

\begin{tabular}{clc}
\hline & \multicolumn{1}{c}{ Parámetro } & Valor, $(\mathbf{m m})$ \\
\hline \multirow{2}{*}{ Rulina } & Diámetro exterior máximo, & 57 \\
primera & Diámetro interno, & 30 \\
operación & Altura, & 26,64 \\
& Profundidad de perfil, & 2,54 \\
& Altura de perfil, & 2,31 \\
\hline \multirow{2}{*}{ Rulina } & Diámetro exterior máximo, & 57 \\
segunda & Diámetro interno, & 30 \\
operación & Altura, & 26,64 \\
& Profundidad de perfil, & 1,32 \\
& Altura de perfil, & 3,49 \\
\hline \multirow{2}{*}{ Mandril } & Diámetro exterior máximo, & 60,60 \\
& Diámetro de base, & 30 \\
\cline { 2 - 3 } & Altura, & 34,65 \\
\hline
\end{tabular}

Análisis de resistencia de los elementos del banco de prueba

El método de elementos finitos (MEF) permite analizar la respuesta de los materiales frente a esfuerzos o cargas específicas, el método tiene muchos campos de aplicación [2125-26]. Dentro de este análisis se

Tabla 2. Propiedades mecánicas de los aceros empleados, librería de SolidWorks ${ }^{\circledR}$.

\begin{tabular}{lcc}
\hline \multicolumn{1}{c}{ Propiedad } & $\begin{array}{c}\text { Acero inoxidable al } \\
\text { cromo }\end{array}$ & $\begin{array}{c}\text { Acero inoxidable } \\
\text { (AISI-304) }\end{array}$ \\
\hline Módulo elástico, $(\mathrm{MPa})$ & 200000 & 190000 \\
Módulo cortante, $(\mathrm{MPa})$ & 77000 & 75000 \\
Densidad, $\left(\mathrm{kg} / \mathrm{m}^{3}\right)$ & 7800 & 8000 \\
Límite de tracción, $(\mathrm{MPa})$ & 413 & 517 \\
Límite elástico, $(\mathrm{MPa})$ & 172 & 206 \\
\hline
\end{tabular}

\section{Selección de cojinetes de rodamiento}

De los resultados del método de elementos finitos se obtienen magnitudes de reacción en las zonas de apoyo que en conjunto con la calculadora de rodamientos del software CAD/CAE [27], permiten elaboran los modelos virtuales, se determinan las condiciones de frontera, se selecciona el material y se configura la densidad de malla. Las propiedades de los materiales utilizados para la evaluación de respuesta mecánica de rulinas y mandril se muestran en la tabla 2. 
e indirectos que se pueden presentar en la fabricación del banco de pruebas. Además, se asigna un margen de utilidad e impuesto de valor agregado sobre los costos calculados.

\section{Resultados y discusión}

Para el diseño conceptual el aspecto económico considera costos por actividades de producción, mantenimiento preventivo y correctivo. El aspecto funcional se relaciona con velocidad rotacional y operaciones para el sellado, finalmente el aspecto de seguridad está enfocado en la prevención de riesgo con la máquina. Las funciones inmersas dentro del banco de pruebas para el cierre de latas se describen a continuación (Tabla 3). La combinación de la columna función y características dan como resultados variantes de diseño (Tabla 4).

Tabla 3. Relación entre funciones y características del banco de pruebas.

\begin{tabular}{|c|c|c|c|c|}
\hline $\mathrm{N} . \stackrel{0}{ }$ & Función & \multicolumn{3}{|c|}{ Característica } \\
\hline & Variantes & 1 & 2 & 3 \\
\hline 1 & Fuente energética & Motor & & \\
\hline 2 & Transmisión de potencia, velocidad & Banc & Piñón & Cadena \\
\hline 3 & Transmisión de potencia, dirección & & & \\
\hline 4 & Accionamiento de rulinas & Neumá & Manua & Leva \\
\hline 5 & Compresión de lata con mandril & Reso & eumátic & \\
\hline 6 & Protección de transmisión & Carca & & \\
\hline
\end{tabular}

Tabla 4. Variantes de diseño conceptual.

\begin{tabular}{cc}
\hline Variante & Combinación \\
\hline 1 & $1.1-2.2-3.1-4.3-5.2-6.1$ \\
2 & $1.1-2.1-3.1-4.2-5.1-6.1$ \\
3 & $1.1-2.3-3.1-4.1-6.1$
\end{tabular}

El diseño que mejor se adapta a los criterios expuestos es la segunda variante con un 50\% aceptación frente a criterios de selección (Tabla $5)$, donde (+) indica la solución a alcanzar, (-) se encuentra fuera del criterio y (/) falta información para considerar la solución como apropiada. La metodología QFD, permitió establecer por medio de aspectos las funciones relevantes para que le banco de pruebas realice la tarea de doble cierre en latas de conservas de atún. 
Tabla 5. Matriz de selección de variante.

\begin{tabular}{|c|c|c|c|c|c|c|c|c|}
\hline 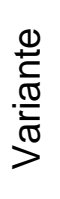 & 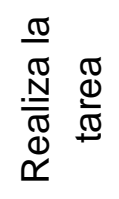 & 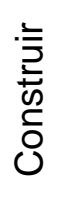 & 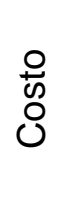 & 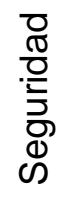 & 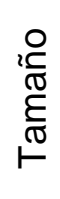 & 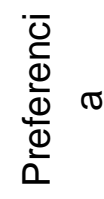 & $\begin{array}{l}\text { I } \\
\text { o }\end{array}$ & $\frac{0^{\circ}}{\frac{\pi}{0}}$ \\
\hline 1 & + & 1 & - & + & + & - & 3 & 30 \\
\hline 2 & + & + & + & + & - & + & 5 & 50 \\
\hline 3 & + & - & 1 & + & - & - & 2 & 20 \\
\hline
\end{tabular}

Banco de pruebas para la evaluación del cierre

El modelo elaborado (Figura 2), se muestra como variante simplificada de las máquinas cerradoras de nivel industrial. La base del modelo

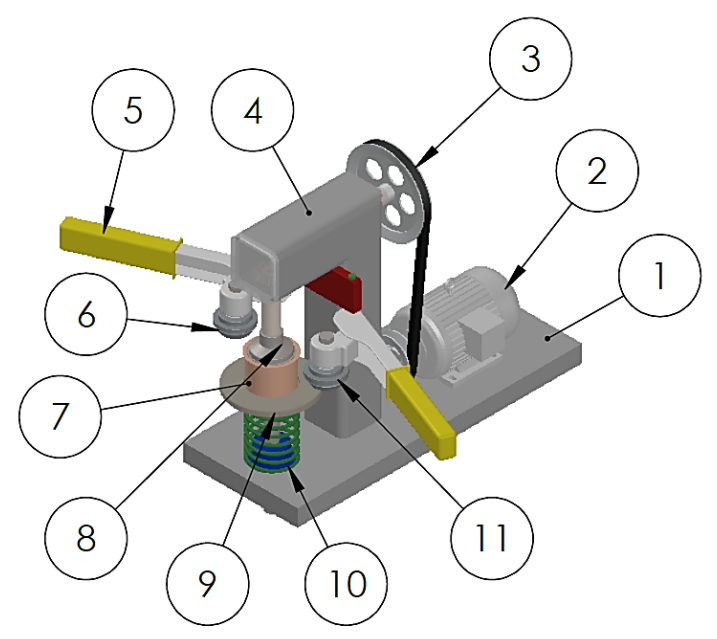

proporciona estabilidad frente a vibraciones y peso propio de los elementos que la conforman. La longitud de la palanca permite suministrar la suficiente presión en el perfil de cierre para ejercer el conformado del metal del envase.

\begin{tabular}{|c|l|}
\hline Comp. & \multicolumn{1}{|c|}{ N. ${ }^{\circ}$ DE PIEZA } \\
\hline 1 & Base de la máquina \\
\hline 2 & Motor eléctrico \\
\hline 3 & Transmisión por correa \\
\hline 4 & Brazo \\
\hline 5 & Palanca \\
\hline 6 & Rulina PO \\
\hline 7 & Lata-tapa \\
\hline 8 & Mandril \\
\hline 9 & Plato compresor \\
\hline 10 & Resorte \\
\hline 11 & Rulina SO \\
\hline
\end{tabular}

Figura 2. Modelo virtual del banco de pruebas.

Transmisión de potencia por banda $\mathrm{V}$

La mayoría de los diseños dependen de la aplicación y las limitaciones de espacios presentes en la maquinaria. La instalación de este tipo de transmisión se torna relativamente fácil además de ser económica, no genera un exceso de ruido y el uso de lubricante se elimina [23-28-29]. De igual forma la selección de bandas y poleas se ve condicionada por un sin número de factores, donde se destaca la potencia de diseño, factor de 
servicio, longitud de banda y ángulo de contacto.

El ajuste de la distancia entre centros en ambas direcciones es lo recomendable, en caso de no existir la posibilidad, es necesario el uso de poleas templadoras. La tabla 6, muestra los resultados relevantes del diseño de la transmisión de potencia por banda. El proceso de cerrado de latas no necesita un alto par torsional, por lo que seleccionó un motor eléctrico de corriente alterna, considerando disponibilidad y economía para desarrollo del prototipo.

Tabla 6. Resumen del diseño de transmisión.

\begin{aligned} & \hline \multicolumn{2}{c}{ Diseño de transmisión por correas $\mathrm{V}$} \\ & Entrada: Motor eléctrico, $1,00 \mathrm{hp} \mathrm{a} 1800 \mathrm{rpm} \\ &$ Factor de servicio: 1,00 \\ & Potencia de diseño: $1 \mathrm{hp} \\ &$ Banda: Sección $3 \mathrm{~V}, 45$ pulgadas de longitud, 1 banda \\ & Relación de velocidades: 4 \\ & Poleas: Motriz, 2,5 pulgadas de diámetro de paso, 1 ranura $3 \mathrm{~V} ; \\ &$ conducida, 10 pulgadas de diámetro de paso, 1 ranura 3V. \\ & Velocidad real de salida: $450 \mathrm{rpm} \\ &$ Distancia entre centros real: 12,106 pulgadas \\ & Ángulo de contacto: $144^{\circ}$, polea menor \\ & Potencia corregida: $0,83 \mathrm{hp}\end{aligned}$

El ángulo de contacto y la longitud de banda son factores que pueden reducir la potencia entregada por el órgano motriz. El ángulo de contacto a su vez tiene que ser $>120^{\circ}$ [23]. La distancia entre centros (C), se encuentra en el intervalo de 25,4 $\mathrm{cm}<\mathrm{C}<95,25 \mathrm{~cm}$, para conservar espacio se utilizó un valor de $\mathrm{C}=30,48 \mathrm{~cm}$. La variable $(\mathrm{C})$ permitió seleccionar una longitud estándar de banda y una distancia entre centros real. Para una reducción adicional de revoluciones y un cambio de dirección vertical para el eje de rotación, se seleccionó un sistema de engranaje corona (36 dientes) y tornillo sin fin de dientes rectos (11 dientes), donde la corona se encuentra conectada de manera directa al mandril. La transmisión de potencia se adquirió y se adaptó a la estructura del banco de pruebas.

\section{Perfiles para efectuar el doble cierre}

Los modelos virtuales responden a las dimensiones reales de las rulinas que se presentó con anterioridad. La zona de trabajo conocida como perfil 
de cierre, es variable y varía de una operación a otra, en dependencia de las características del ala y espesor que se somete a la operación de doble cierre, (Figura 3). A medida que incrementa el tamaño y espesor del ala, también aumenta la profundidad y altura de los perfiles.
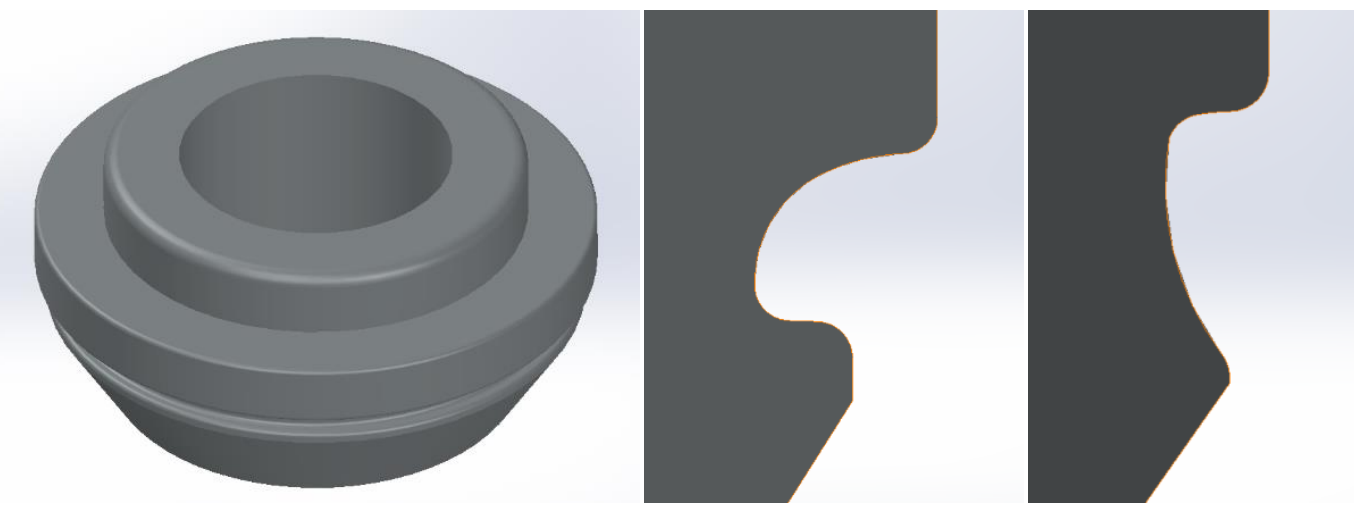

Figura 3. Modelo virtual de rulina, perfiles elaborados para la primera y segunda operación.

Las rulinas de primera y segunda operación se alinean con una distancia de separación medido desde su punto de control y la superficie superior del labio del mandril a $0,08 \mathrm{~mm}$ y $0,13 \mathrm{~mm}$ respectivamente. Esta alineación que puede variar evita un contacto directo del perfil con el labio del mandril que dañaría la sección de trabajo de la rulina.

Un ejemplo de esfuerzo de compresión para un espesor de lata frecuente de 0,18 $\mathrm{mm}$ son 200 libras [24]. La presión que ejerce cada una de las rulinas en las operaciones $1 \mathrm{y}$ 2 determina la calidad de cierre de la lata. El desarrollo del banco de pruebas permite realizar varias configuraciones de cierre analizando la distancia entre centro de los elementos mandril y rulinas.

Tensiones, deformaciones en rulinas y mandril

Las máximas tensiones (von Mises) en el mandril alcanzan valores de 15,6 MPa producto de la fuerza perpendicular de $890 \mathrm{~N}$ que se transmite del plato base a través del conjunto lata-tapa (Figura 4). Las tensiones promedias que se distribuyen en el labio del mandril se encuentran en el rango de 7,8 MPa a 10,92 MPa. Por otro lado, las deformaciones máximas registrados son de 0,000051 $\mathrm{mm}$ también presentes en la superficie de contacto con el material de la lata. 

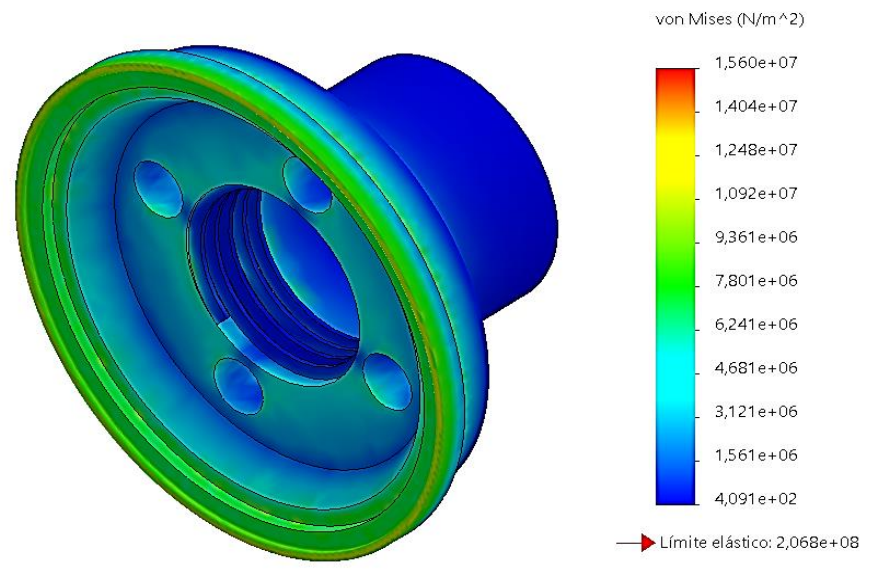

Figura 4. Distribución de tensiones en el mandril.

Las rulinas que presentan una sujeción de rodamiento no se someten a niveles elevados de presión. Para observar cómo se distribuye una fuerza a lo largo del perfil se utilizó un máximo de $89 \mathrm{~N}$ que es significativamente elevada. Como resultado, las tensiones máximas en el perfil de cierre para la primera rulina son de 0,3336 MPa y para la segunda de 0,2011 MPa (Figura 5). Las deformaciones máximas que se pueden alcanzar en el perfil son de $0,00000175 \mathrm{~mm}$ y de $0,0000007 \mathrm{~mm}$ respectivamente.
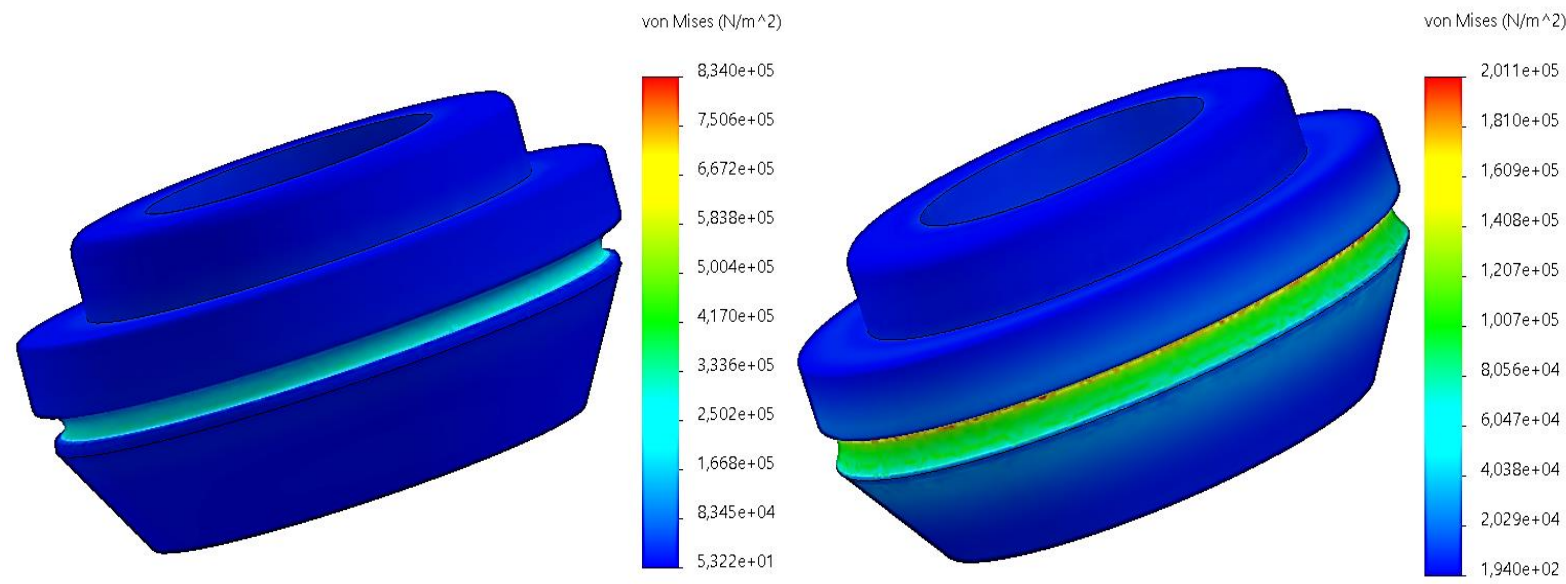

Figura 5. Distribución de tensiones en rulinas de primera y segunda operación.

La distribución del factor de seguridad a través de la pieza por el criterio de tensiones de von Mises indica que el valor mínimo de este parámetro es de $8,569 \cdot 10^{\wedge} 2$ (Figura 6), la poca deformación que se puede presentar en las superficies sometidas a esfuerzos y las tensiones no comprometen la funcionalidad de los elementos, pues no superan el límite elástico del material. 

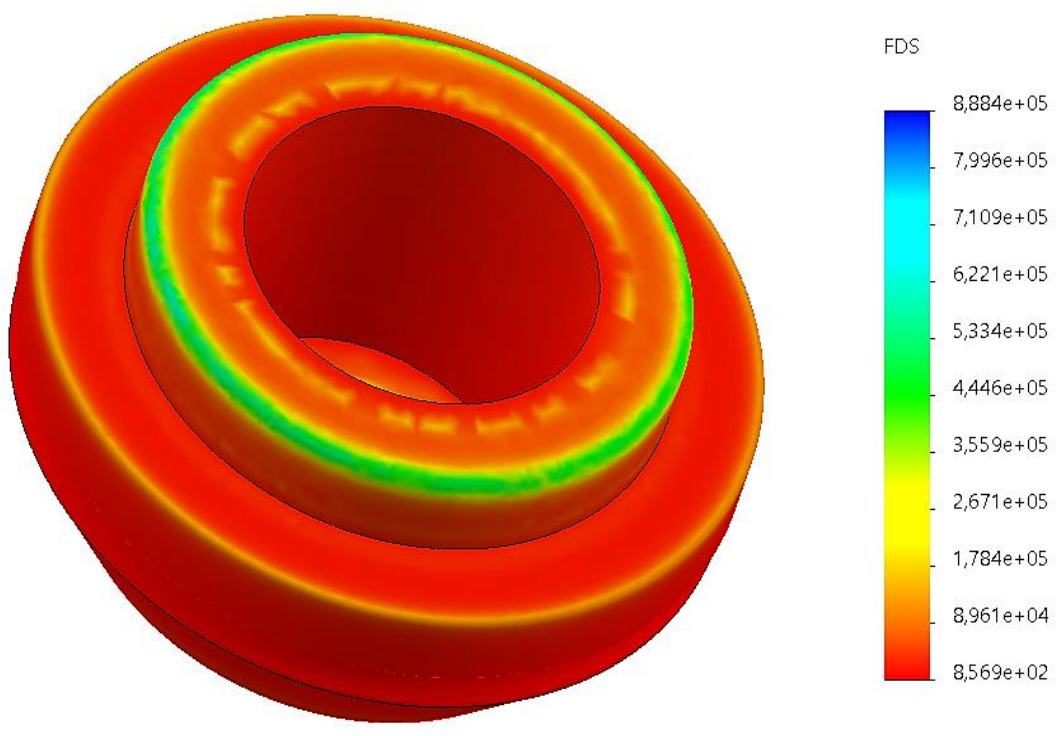

Figura 6. Distribución del factor de seguridad.

\section{Selección de rodamientos}

Cada rulina se monta sobre rodamientos que se seleccionan a partir de los resultados de las fuerzas del conector de tipo rodamiento. La fuerza axial y cortante resultante son $7,901 \mathrm{~N}$ y $0,0004 \mathrm{~N}$ respectivamente, la presencia de ambas fuerzas condiciona seleccionar un rodamiento de bolas con contacto angular de una hilera (DIN 7200B), que además de soportar grandes cantidades de cargas axial y radial, reduce la necesidad de lubricación. Con un intervalo de confiabilidad del $95 \%$, la vida en horas en función de las fuerzas y velocidad de operación es de $3,819 \cdot 10^{\wedge} 10 \mathrm{hrs}$.

\section{Desarrollo y puesta punto del banco de pruebas}

La construcción del banco de pruebas contempló algunas etapas, en primera instancia se realiza la adquisición de elementos, como rulinas, mandril, poleas, correas, rodamientos, motor eléctrico, entre otros. La fabricación comenzó por la base de la máquina y la transmisión de potencia, posteriormente la colocación de palancas con sus respectivas rulinas y finalmente la disposición del plato base alineado con el mandril (Figura 7). 


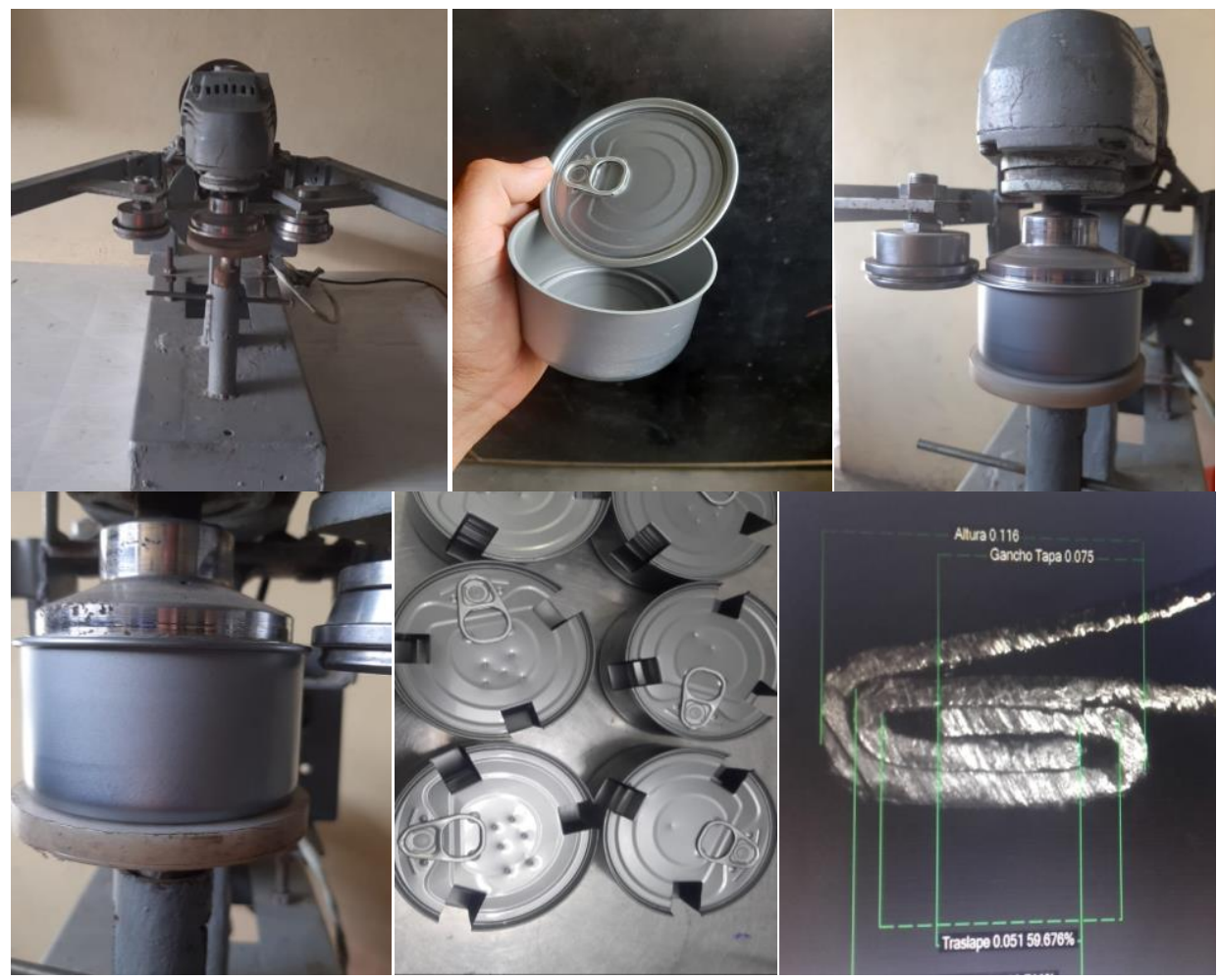

Figura 7. Banco de pruebas desarrollado y proceso de cierre.

El proceso de cierre se realiza de manera asistida. El motor eléctrico de la unidad suministra el torque para producir el giro en el mandril por medio de las transmisiones de potencia. El plato base aloja el conjunto lata-tapa y ejerce presión en el mandril con ayuda de un resorte de amortiguación. La presión en este punto se logra gracias a la altura de la lata $(50,80 \mathrm{~mm})$ que supera el espacio libre entre el mandril y el plato. El envase de conserva se mantiene en movimiento rotacional constante durante todo el proceso, la primera rulina gira en dirección opuesta al entrar en contacto con el envase y deforma el material de ambas pestañas creando un enganche por medio del perfil de engargolado, esta operación genera también un pequeño planchado que será aprovechado por la segunda rulina. La segunda operación plancha el enganche y distribuye el compuesto de sellado en las cavidades del cierre, evitando así filtraciones de fluidos del exterior al interior del envase y viceversa. Ambos rulinas se encuentran montadas sobre rodamientos de rodillos cilíndricos por la presencia de cargas radiales y axiales. Finalmente se realizan cortes de sección a las latas y se evalúa por medio de imagen 
digitalizadas la calidad del cierre realizado.

\section{Análisis económico del banco de pruebas}

Se realizó el cálculo de los gastos procedentes de la adquisición de materiales y equipo, donde se destacan las rulinas, el mandril y el motor eléctrico que representan el mayor porcentaje de costo. De manera adicional se analizan otros gastos asociados a la construcción del banco de pruebas que permiten establecer un valor real para la fabricación del prototipo (Tabla 7).

Tabla 7. Ficha de costo del banco de pruebas.

\begin{tabular}{lcc}
\hline Concepto & Fila & Valor (USD) \\
\hline Materiales y equipo & 1 & 443 \\
Construcción mecánica & 2 & 90 \\
Gasto de elaboración & 3 & 533 \\
Fuerza de trabajo & 4 & 40 \\
Gastos directo (3+4) & $\mathbf{5}$ & $\mathbf{5 7 3}$ \\
Gastos varios (14\% de 5) & 6 & 80,22 \\
Gastos indirectos (5+6) & $\mathbf{7}$ & $\mathbf{6 5 3 , 2 2}$ \\
Margen de utilidad (15\% de 5) & 8 & 85,95 \\
Precio de la unidad (7+8) & 9 & 739,17 \\
IVA 12\% & 10 & 88,70 \\
Total & $\mathbf{1 1}$ & $\mathbf{8 2 7 , 8 7}$ \\
\hline
\end{tabular}

\section{Conclusiones}

El banco de pruebas desarrollado es capaz de reproducir el procedimiento de cierre de latas de conservas de alimentos que se realiza con las máquinas industriales, así como el régimen de operación y regulación.

A partir del diseño conceptual, se identificó la variante de diseño del banco más significativa que cumple las características funcionales requeridas para llevar a cabo la operación de cierre de latas circulares de conserva, en condiciones controladas.

El análisis de elementos finitos permitió evidenciar que las deformaciones y tensiones que actúan sobre el mandril y rulinas durante el sellado de las latas, no comprometen su integridad estructural, mostrando un factor de seguridad que para las rulinas es muy elevado. 


\section{Bibliografía}

[1] Guarda T., Díaz-Nafría J.M., Augusto M.F., and Vitor J.A.: Territorial intelligence in the impulse of economic development initiatives for artisanal fishing cooperatives, Springer, 2018, 105-115.

[2] Lucas F.S.: La calidad y competitividad en las empresas de la ciudad de Manta-Ecuador. Mikarimin. Revista Científica Multidisciplinaria, Vol. 3, No. 3 (2017) 09-18.

[3] Acosta M.R.: Ejes centrales de acumulación de capital en el Ecuador actual: una panorámica general. Revista Economía, Vol. 70, No. 111 (2018) 77-93.

[4] Bedoya J.L.P.: Aspectos Biológicos y Pesqueros del Atún Aleta Amarilla Thunnus albacares Capturado por la Flota Atunera Cerquera Ecuatoriana, Período 20092013 (2014)

[5] Arteaga-Linzan Á.R.: EnergyEconomic Evaluation in the Production of Canned Tuna in Ecuadorian Industry. Revista Ciencias Técnicas Agropecuarias, Vol. 26, No. 3 (2017) 94-102.

[6] Campling L.: Trade politics and the global production of canned tuna. Marine Policy, Vol. 69 (2016) 220-228.
[7] García-del-Hoyo J.J., Jiménez-Toribio R., and Guillotreau P.: A demand analysis of the Spanish canned tuna market. Marine Policy, Vol. 86 (2017) 127133.

[8] DeBeer J., Nolte F., Lord C.W., and Colley J.: Precooking Tuna: A Heat of Summation Analysis of Different Heating Profiles. Food Protection Trends, Vol. 39, No. 2 (2019) 127-136.

[9] Montanari A., Barone C., Barone M., and Santangelo A.: Canned Tomato Sauces and Beans: Industrial Processes, Springer, 2018, 43-53.

[10] Ozkan G., Franco P., De Marco I., Xiao J., and Capanoglu E.: A review of microencapsulation methods for food antioxidants: Principles, advantages, drawbacks and applications. Food chemistry, Vol. 272 (2019) 494-506.

[11] Cova Caiazzo F., Brambilla L., Montanari A., and Mischler S.: Chemical and morphological characterization of commercial tinplate for food packaging. Surface interface analysis, Vol. 50, No. 4 (2018) 430-440.

[12] Li X.: Metal food packaging design based on hazard analysis critical control point (HACCP) system in canned 
food safety. Acta Universitatis Cibiniensis. Series E: Food Technology, Vol. 20, No. 1 (2016) 93-104.

[13] Xie J., Tang Y., Yang S.-P., and Qian Y.-F.: Effects of whey protein films on the quality of thawed bigeye tuna (Thunnus obesus) chunks under modified atmosphere packaging and vacuum packaging conditions. Food science biotechnology, Vol. 26, No. 4 (2017) 937-945.

[14] Pino Hernández E.J.G., Serrada A., and Farías C.: Efecto del proceso de esterilización en conservas de atún al natural. Saber, Vol. 29 (2017) 374-384.

[15] Lizarraga Vargas P.E.: Evaluación de parámetros para el procesamiento de conserva de pejerrey (Odontesthes regia) en tres líquidos de gobierno, Universidad Nacional de San Agustín de Arequipa, 2018.

[16] Miranda Jara A.Y., Miranda Chávez A.H., and Chuquilín Goicochea R.C.: Physicochemical and sensorial evaluation of chicken gizzards (Gallus gallus) in soy sauce canned. Biotecnología en el Sector Agropecuario y Agroindustrial, Vol. 18, No. 1 (2020) 35-45.

[17] Hernández G.L.-G.: Ensayos de compresión sobre latas de refresco. Análisis de tensiones y deformaciones, Universidad Politécnica de Madrid, 2018.

[18] Pascual P.A.L., and Pascual G.L.: Process Optimization, Consumer testing and Shelflife Determination of Canned "Halang-halang": A Filipino Traditional Food. International Journal of Environmental Agriculture Research, Vol. 4, No. 2 (2018) 40-46.

[19] Brambilla L., Caiazzo F.C., Michel A., Mischler S., and Bertholon R.: Degradation of heritage cans: Monitoring of museums' collections. Measurement, Vol. 127 (2018) 256-263.

[20] Dantas F.B.H., and Dantas S.T.: Canned food and packaging parameters. Chapter, Reference Module Food Science (2016) 1-5.

[21] Montes C., Herrera M., and Guerrero E.S.: Dispositivo para la movilidad autónoma de personas usuarias de sillas de ruedas convencionales. Revista Ingeniería Biomédica, Vol. 13, No. 25 (2019) 13-24.

[22] Sánchez J.G.L., Grajales M.J., and Aguilar J.S.: Despliegue de la Función de Calidad para el Diseño de Limpiador Automático de Rodillo. Conciencia Tecnológica, No. 52 (2016) 51-54.

[23] Mott R.L.: Diseño de Elementos de Máquinas, 
Pearson educación, 2006, 264-282.

[24] Valderas A., "Envases metálicos," 2017.

[25] Alves Filho A.: Elementos Finitos a base da Tecnologia CAE, Saraiva Educação SA, 2018

[26] Medina R., Salas M., Luco R., and Bertram V.: Análisis de Estructuras Navales mediante el método de elementos finitos. Síntesis Tecnológica, Vol. 2, No. 1 (2017) 27-36.

[27] Garcés Núñez R.G., and Morocho Polo S.J.: Diseño de una máquina ordeñadora accionada por una fuerza no convencional, Universidad Señor de Sipán, 2018.

[28] Duque H.D.P., Posada L.M.L., and Angulo E.S.M.: Metodología del diseño y modelado de un accionamiento mecánico. Scientia et technica, Vol. 22, No. 1 (2017) 24-33.

[29] Frendo F., and Bucchi F.: Enhanced brush model for the mechanics of power transmission in flat belt drives under steady-state conditions: Effect of belt elasticity. Mechanism Machine Theory, Vol. 153 (2020) 103998. 